\title{
Collision Based Adaptive Time Synchronization Verification Method for Ocean Sensor Networks
}

\author{
Ying Guo ${ }^{\mathrm{a}}$, Haiwei Cui \\ Qing Dao University of Science and Technology, Qing Dao, China \\ aguoying@qust.edu.cn
}

Keywords: Ocean sensor networks, Time synchronization, Collision, Parameter adaptive

\begin{abstract}
Time synchronization is a critical issue for ocean sensor networks, existing time synchronization algorithms focus on the calculation of clock skew and offset, the studies on time synchronization verification is limited. We propose a collision based adaptive time synchronization verification method for ocean sensor networks. It makes use of information collision in underwater channel to discover unsynchronized nodes, and takes ocean environmental parameter adaptive algorithm to improve computation precision. Simulation results show that our design performs much better than existing time synchronization verification schemes
\end{abstract}

\section{Introduction}

Ocean sensor networks are widely used in environment supervision, undersea exploration, disaster prevention, target tracking, security surveillance, and so on [1,2]. In most applications, all sensor nodes must have a common time scale to coordinate and collaborate with each other. Finding out unsynchronized nodes to do time synchronization is an essential service for ocean sensor networks. We call this problem as time synchronization verification in this paper.

In the past few years, many time synchronization protocols have been proposed for underwater sensor networks [3], such as, TSHL [4], MU-Sync [5], Mobi-Sync [6] and D-sync [7]. These algorithms make great efforts to reduce the bad influence of clock skew and offset, but none of them is designed for time synchronization verification.

In order to fill this gap, we propose a collision based adaptive time synchronization verification method for ocean sensor networks. Coll-Sync [8] shows that collision contains highly useful information that can be leveraged to improve time synchronization greatly. In this paper, we utilize collision to discover unsynchronized nodes, and adopt parameter adaptive algorithm to adapt ocean environment.

\section{Algorithm Design}

As shown in figure 1, one beacon node S with standard time and three common nodes A, B and C are deployed in ocean environment, all of them keep still. Node S sends the synchronization message at time $T_{1}$. The packet contains the next receiving time $T_{\mathrm{S}}$, and the sending time $T_{1}$ which is a timestamp of MAC layer. The message arrives at node $\mathrm{A}, \mathrm{B}$ and $\mathrm{C}$ at their local time $T_{1 \mathrm{~A}}, T_{1 \mathrm{~B}}$ and $T_{1 \mathrm{C}}$ with propagation delay $t d_{\mathrm{A}}, t d_{\mathrm{B}}$ and $t d_{\mathrm{C}}$ separately. We could obtain Eq.1,

$$
\left\{\begin{array}{l}
t d_{A}=T_{1 A}-T_{1} \\
t d_{B}=T_{1 B}-T_{1} \\
t d_{C}=T_{1 C}-T_{1}
\end{array}\right.
$$

Assuming the message propagation delays of go and back are equal, nodes A, B and C send respond message to node $S$ at their local time $T_{S}-t d_{A}, T_{S}-t d_{B}$ and $T_{S}-t d_{C}$. If these nodes have been synchronized exactly, the messages of nodes $\mathrm{A}, \mathrm{B}$ and $\mathrm{C}$ arrive at node $\mathrm{S}$ at the same time. In this situation, node $S$ could not receive any effective information as these messages collided at its receiving terminal. Conversely, if one of these nodes has not been synchronized exactly, node S could 
receive some message from this node. In this way, the node needed time synchronization be discovered.

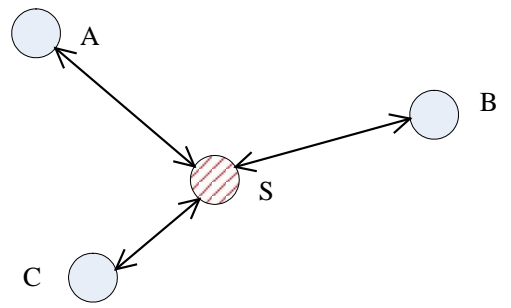

Fig. 1 Network model

In order to improve verification precision in real ocean environment, a collision based ocean environmental parameter adaptive time synchronization verification method is proposed for mobile ocean sensor networks.

Underwater sensor nodes equipped with various kinds of sensors to monitor ocean environment, such as temperature, salinity, pressure and so on, they could also perceive their locations by existing location methods [9-10]. Take figure 1 for example, one fixed beacon node $\mathrm{S}$ with standard time and three common nodes $\mathrm{A}, \mathrm{B}$ and $\mathrm{C}$ are deployed, these common nodes could move with ocean current. Node $\mathrm{S}$ sends a synchronization message. The packet contains its position $\left(X_{\mathrm{S}}, Y_{\mathrm{S}}, Z_{\mathrm{S}}\right)$, temperature $t_{\mathrm{S}}$, salinity $s_{\mathrm{S}}$, pressure $p_{\mathrm{S}}$, the next receiving time $T_{\mathrm{S}}$ and sending time $T_{1}$ which comes from the timestamp of MAC layer.

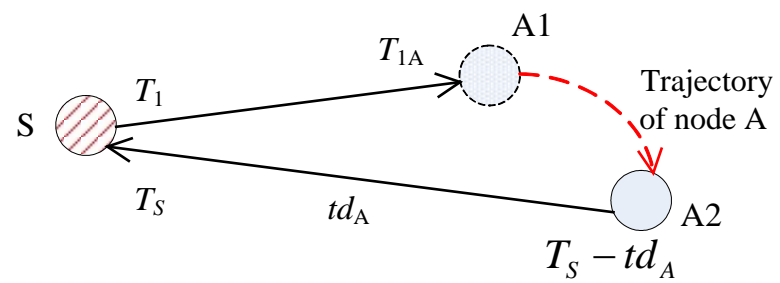

Fig. 2 Node mobility

Node A receives the synchronization message at position A1, as in figure 2. It waits several seconds to send the response information. During the waiting time, node A moves with ocean current to position A2. The waiting time could be computed,

$$
T_{S}-\left(T_{1 A}-T_{1}\right)+\Delta T
$$

In which, $\Delta T$ is the computation margin decided in advance.

The distance from node $A$ to node $S$ could be computed based on node A's current position A2 $\left(X_{\mathrm{A}}, Y_{\mathrm{A}}, Z_{\mathrm{A}}\right)$ and node $\mathrm{S}^{\prime}$ position $\left(X_{\mathrm{S}}, Y_{\mathrm{S}}, Z_{\mathrm{S}}\right)$, as shown in Eq.2,

$$
d_{A S}=\sqrt{\left(X_{S}-X_{A}\right)^{2}+\left(Y_{S}-Y_{A}\right)^{2}+\left(Z_{S}-Z_{A}\right)^{2}}
$$

At the same time, node A measures its current temperature $t_{\mathrm{A}}$, salinity $s_{\mathrm{A}}$, and pressure $p_{\mathrm{A}}$. Underwater acoustic velocity varieties with temperature $t\left({ }^{\circ} \mathrm{C}\right)$, salinity $s(\%)$ and pressure $p\left(\mathrm{~kg} / \mathrm{cm}^{2}\right)$, Wilson equation [11] is the most widely used empirical formula:

$c=1449.30+\Delta c_{t}+\Delta c_{z}+\Delta c_{p}+\Delta c_{t s p}$

in which,

$$
\begin{aligned}
& \Delta c_{t}=4.587 t-5.356 \times 10^{-2} t^{2}-2.604 \times 10^{-4} t^{5} \\
& \Delta c_{z}=1.19(s-35)+9.6 \times 10^{-2}(s-35)^{2} \\
& \Delta c_{p}=1.5848 \times 10^{-1} p+1.572 \times 10^{-5} p^{2}-3.46 \times 10^{-12} p^{4} \\
& \Delta c_{t s p}=1.35 \times 10^{-5} t^{2} p-7.19 \times 10^{-7} t^{2}-1.2 \times 10^{-2}(s-35) t
\end{aligned}
$$

Based on Eq.3, the propagation speed between node A and $S$ is shown in Eq.4, 


$$
c_{A S}=\int_{p_{S}}^{p_{A}} \int_{S_{S}}^{s_{A}} \int_{t_{S}}^{t_{A}}\left(1449.30+\Delta c_{t}+\Delta c_{z}+\Delta c_{p}+\Delta c_{t s p}\right) d p d s d t
$$

The information propagation delay $t d_{A}$ from node A to node $S$ could be obtained by distance formula of Eq.5,

$$
t d_{A}=\frac{d_{A S}}{c_{A S}}
$$

Node A sends respond information to node $\mathrm{S}$ at its local time $T_{S}-t d_{A}$. In the similar way, node B and $C$ send their respond messages to node $S$ at their local time $T_{S}-t d_{B}$ and $T_{S}-t d_{C}$.

In our design, all the sensor nodes' clock deviations are different. So the information of unsynchronized nodes could not collide at the receiving terminal. In this situation, node $S$ could not receive any effective information at time $T_{\mathrm{S}}$ means these nodes have been synchronized exactly. As the messages of nodes $\mathrm{A}, \mathrm{B}$ and $\mathrm{C}$ arrive at node $\mathrm{S}$ at the same time and collide at node S's receiving terminal. Node $S$ could receive some message from one of these nodes means this node is unsynchronized and needs time synchronization again.

Due to the influence of non-stationary ocean environment, running only one time of synchronization verification algorithm could not determine if a node's clock is unsynchronized. Therefore, running the validation algorithm several times, when the probability of one node's message does not collide with other nodes is higher than a predetermined limit value, this node is identified as an unsynchronized node and needs time synchronization again.

\section{Simulation}

In the simulation, a fixed beacon node with standard time and several common nodes are deployed in ocean environment. Some of these common nodes have been synchronized exactly, and others are unsynchronized with different clock deviations from $0.1 \mathrm{~s}$ to $4.8 \mathrm{~s}$. We compare our design with traditional method (TM) to prove its performance. In traditional method, the beacon node sends a synchronization message includes its sending time comes from MAC layer timestamp. The node receives this packet and computes the difference of receiving time and sending time. The node needs synchronization only when the difference bigger than a predefined value, which is set as $500 \mathrm{~ms}$ in the following simulations.

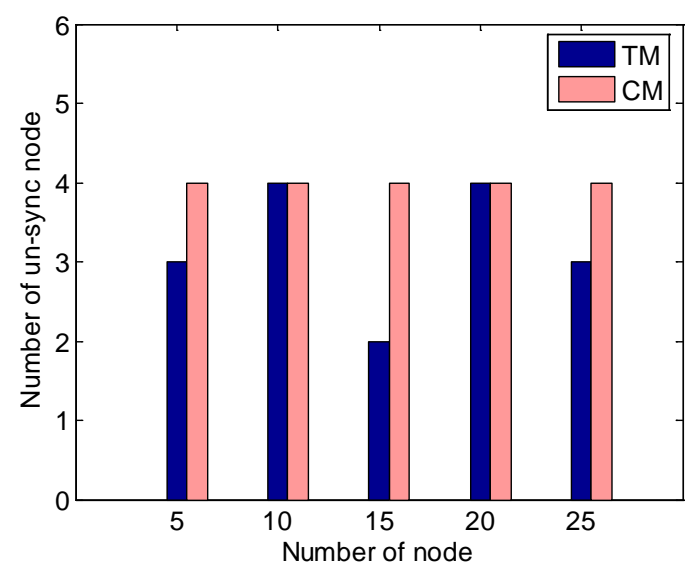

(a)

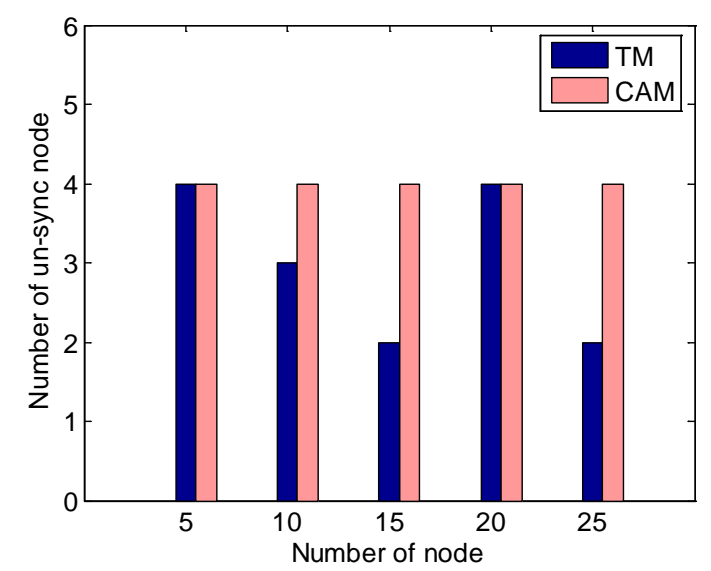

(b)

Fig. 3 Number of unsynchronized nodes (a) in static networks (b) in mobile networks

The number of deployed common nodes added from five to twenty-five, in which four nodes are unsynchronized. The number of unsynchronized nodes discovered by collision based time synchronization verification method (CM) and traditional method (TM) is shown in figure 3(a) and 3(b). TM could not discover all the unsynchronized nodes, it displays in mobile environment worse than in static environment. As node mobility aggravates the variation of propagation delay and influences the precision of TM. CAM has higher accuracy and finds every unsynchronized node, 
because the ocean environmental parameter adaptive algorithm compensates the influence of node mobility.

\section{Conclusions}

We present a collision based adaptive time synchronization verification method for ocean sensor networks. It could be used in both static and mobile ocean sensor networks to discover unsynchronized nodes.

\section{Acknowledgments}

This work was supported in part by the National Natural Science Foundation of China under Grant No. 61103196, 61170258 and 61379128. Research Award Fund for Excellent Young Scientist of Shandong Province under Grant No. BS2012DX011. Qingdao Science and Technology Development Program under Grant No. 12-1-4-3-(16)-jch.

\section{References}

[1] Y.F. Wang, Z.C. Zhang and G.G. Bi, 17th International Conference on Telecommunications, (Doha, April 4-7, 2010), p.779-783.

[2] R. Khan, Q. Gang, W. Wang: Applied Mechanics and Materials, Vol.321-324 (2013), p.2868-2876.

[3] J. Liu, Z.H. Wang, J.Z. Peng, M. Zuba, J.H. Cui and S.L. Zhou, IEEE Global Telecommunications Conference (GLOBECOM), (Houston, TX, USA, December 5-9, 2011), p.1-6.

[4] A.A. Syed, J.Heidemann: 25th IEEE International Conference on Computer Communications (INFOCOM), (Barcelona, Spain, April, 2006), pp1-12.

[5] N. Chirdchoo, W.S. Soh and K.C. Chua: Proceedings of WUWNet, (New York, NY, USA, September, 2008), p.35-42.

[6] J. Liu, Z. Zhou, Z. Peng and J.H. Cui: IEEE Transactions on Parallel and Distributed Systems, Vol.24, (2012), No.2, p.406-416.

[7] F. Lu, D. Mirza and C. Schurger: The Fifth ACM International Workshop on Underwater Networks (WUWNET), (Woods Hole, MA., September, 2010).

[8] Y. Guo and Y.T. Liu: Applied Mechanics and Materials Journal, Vols.246-247 (2012), p.806-810.

[9] X.Y. Wei, C. Zhu and S.D. Qiao: Advanced Materials Research, Vol.989-994 (2014), p.169-4174.

[10]Y. Guo, and Y.T. Liu: Computers and Electrical Engineering, Vo.39, (2013) No.6, p.1812-1821.

[11]M. Stojanovic: Proc. ACM WUWNet, (Los Angeles, CA, September, 2006), p.41-47. 